\title{
BMJ Open Paediatric postdischarge mortality in developing countries: a systematic review
}

\author{
Brooklyn Nemetchek, ${ }^{1}$ Lacey English, ${ }^{2}$ Niranjan Kissoon, ${ }^{3,4}$ \\ John Mark Ansermino, ${ }^{4,5}$ Peter P Moschovis, ${ }^{6}$ Jerome Kabakyenga, ${ }^{7}$ \\ Susan Fowler-Kerry, ${ }^{1}$ Elias Kumbakumba, ${ }^{8}$ Matthew O Wiens ${ }^{4,8}$
}

To cite: Nemetchek B, English L, Kissoon N, et al. Paediatric postdischarge mortality in developing countries: a systematic review. BMJ Open 2018;8:e23445. doi:10.1136/ bmjopen-2018-023445

\section{- Prepublication history and} additional material for this paper are available online. To view these files, please visit the journal online (http://dx.doi org/10.1136/bmjopen-2018023445).

Received 9 April 2018 Revised 24 July 2018 Accepted 24 September 2018

Check for updates

(c) Author(s) (or their employer(s)) 2018. Re-use permitted under CC BY-NC. No commercial re-use. See rights and permissions. Published by BMJ.

For numbered affiliations see end of article.

Correspondence to Dr Matthew 0 Wiens; mowiens@outlook.com

\section{ABSTRACT}

Objectives To update the current evidence base on paediatric postdischarge mortality (PDM) in developing countries. Secondary objectives included an evaluation of risk factors, timing and location of PDM.

Design Systematic literature review without meta-analysis. Data sources Searches of Medline and EMBASE were conducted from October 2012 to July 2017.

Eligibility criteria Studies were included if they were conducted in developing countries and examined paediatric PDM. 1238 articles were screened, yielding 11 eligible studies. These were added to 13 studies identified in a previous systematic review including studies prior to October 2012. In total, 24 studies were included for analysis.

Data extraction and synthesis Two independent reviewers extracted and synthesised data using Microsoft Excel. Results Studies were conducted mostly within African countries (19 of 24) and looked at all admissions or specific subsets of admissions. The primary subpopulations included malnutrition, respiratory infections, diarrhoeal diseases, malaria and anaemia. The anaemia and malaria subpopulations had the lowest PDM rates (typically $1 \%-2 \%$ ), while those with malnutrition and respiratory infections had the highest (typically 3\%-20\%). Although there was significant heterogeneity between study populations and follow-up periods, studies consistently found rates of PDM to be similar, or to exceed, in-hospital mortality. Furthermore, over two-thirds of deaths after discharge occurred at home. Highly significant risk factors for PDM across all infectious admissions included HIV status, young age, pneumonia, malnutrition, anthropometric variables, hypoxia, anaemia, leaving hospital against medical advice and previous hospitalisations.

Conclusions Postdischarge mortality rates are often as high as in-hospital mortality, yet remain largely unaddressed. Most children who die following discharge do so at home, suggesting that interventions applied prior to discharge are ideal to addressing this neglected cause of mortality. The development, therefore, of evidence-based, risk-guided, interventions must be a focus to achieve the sustainable development goals.

\section{INTRODUCTION}

The third of 17 United Nations sustainable development goals (SDGs) emphasises preventable deaths of newborns and children under 5 years of age, with all countries aiming to reduce under-5 mortality to at least

\section{Strengths and limitations of this study}

- Extensive literature search of Medline and Embase with independent screening of all abstracts and eligible full-text publications by two investigators.

- Extensive data extraction on risk factors for mortality within each study population.

- Few studies were prospective and focused on measurement of post-discharge mortality as a primary outcome.

- Heterogeneity in populations, duration of follow-up and high proportion of loss to follow-up may limit the external validity and underestimate outcome rates.

- No optimal method to assess the risk of bias in included studies.

as low as 25 deaths per 1000 live births by the year 2030. ${ }^{1}$ Although significant progress was made during the Millennium Development Goal era (1990-2015), preventable childhood deaths remain high in Southern Asia and sub-Saharan Africa. ${ }^{2}$ These deaths result largely from infectious diseases (including malaria, pneumonia, diarrhoea, etc), which lead to sepsis. ${ }^{3}$ Children are particularly vulnerable in the months following hospital discharge, with a growing body of research demonstrating that postdischarge deaths occur in similar numbers as during hospital admission. Despite the staggering burden of postdischarge mortality, this issue has been largely neglected when examining paediatric mortality from infectious disease. The 2017 United Nations World Health Assembly (WHA) resolution calling for improvement in prevention, diagnosis and management of sepsis is timely as it emphasises the need for improved follow-up care, particularly for developing countries, within their recommended actions for reducing the burden of sepsis globally. ${ }^{4}$ Member states are urged to emphasise the impact of sepsis on public health, of which postdischarge mortality is a crucial aspect. ${ }^{2}$ Thus, as the international community works towards achieving the WHA resolution and the third SDG, addressing the 
burden of paediatric postdischarge mortality is of utmost importance.

A systematic literature review conducted in 2012 examined the burden of paediatric postdischarge mortality in resource-poor countries. ${ }^{5}$ This systematic review found that the rate of paediatric postdischarge death is often as high as in-hospital mortality rates, with two-thirds of these deaths occurring outside the health system, usually at home. Common risk factors for postdischarge mortality included young age, malnutrition, HIV, pneumonia and recent prior admissions.

Despite the high burden of postdischarge death, this issue continues to receive insufficient recognition at either national or international levels. The lack of research and data highlighting the burden of postdischarge mortality relegates care following discharge as a low priority to policy makers. Additional studies published since the last systematic review contribute to the growing evidence base that can galvanise both researchers and policy makers to action.

The purpose of this systematic review, therefore, is to update the literature addressing the critical nature of paediatric postdischarge mortality in resource-poor settings, propelling research and interventions towards the goal of reduced child mortality.

\section{METHODS}

\section{Objective and study eligibility criteria}

The primary objective was to determine the risk factors and rates of mortality in children following discharge from hospitals in developing countries. Table 1 outlines the study inclusion eligibility, determined through the Population, Interventions, Comparisons, Outcomes and Study Design format.

\section{Patient and public involvement}

Patients and the public were not involved in the design or conduct of this study.

\section{Search strategy}

Articles published and indexed between 1 January 2012 and 18 July 2017 were identified using the MEDLINE and EMBASE databases within the OVID platform. The detailed search strategy for each database is outlined in online supplementary appendix 1 . Studies conducted prior to 2012 were identified from a prior publication, using a similar search strategy. ${ }^{5}$ Articles were included if the study was conducted in a developing country (defined as countries currently (2016) classified by the United Nations Development Programme as having a low Human Development Index plus those countries

Table 1 Population, Interventions, Comparisons, Outcomes and Study Design

Population Paediatric patients discharged from hospitals in developing countries, as defined as those countries currently (2016) classified by the United Nations Development Programme as having a low Human Development Index plus those countries included previously (2011) as having a low Human Development Index. ${ }^{67}$

Exclusion criteria:

- No paediatric data or paediatric data not differentiated from adult populations.

- No postdischarge information or patients not discharged from a hospital setting.

- Discharge was following a non-admission (eg, following birth).

- Studies representing a specific non-infectious disease population where postdischarge care and outcomes would likely be different than that following acute (primarily infectious) illness including:

- Surgical population;

- Specific congenital disease (cardiac, renal, etc);

- Cancer;

- Specific non-infectious admission including trauma, kidney disease, cardiac disease, ophthalmic disease, sickle cell disease, liver disease, epilepsy, burns, poisoning, asthma, etc.

- Study was unpublished, published only in abstract form or in a language other than English, or provided no original data.

$\begin{array}{ll}\text { Interventions } & \text { Studies may or may not include an interventional arm (ie, both arms of an RCT will be included). } \\ \text { Comparisons } & \text { NA } \\ \text { Outcomes } & \text { Primary outcome: } \\ & \text { Secondary outcomes: } \\ & \text { Pn-hospital mortality; } \\ & \text { Risk factors for postdischarge mortality. } \\ \text { Study design } & \text { Eligible study designs include the following: } \\ & \text { Randomised controlled trials (RCTs); } \\ & \text { Prospective or retrospective cohort studies; } \\ & \text { Studies using surveillance data; } \\ & \text { Study designs which include a population discharged and then followed up (including case-control, } \\ & \end{array}$

NA, not applicable. 
included previously (2011) as having a low Human Development Index ${ }^{67}$ ), included children admitted to hospital for medical reasons, and included follow-up to capture vital status during the postdischarge period. ${ }^{56}$ Furthermore, references of all included articles were reviewed to identify other potentially eligible studies not captured in the systematic search.

\section{Study selection and data extraction}

Two investigators (BN, LE) independently screened articles during two rounds of review. The first round consisted of reviewing all abstracts for the presence of specific exclusion criteria. The second round of review consisted of a detailed review of remaining articles in full-text format. In both rounds, any discrepancies were resolved through discussion and consensus. A third investigator (MOW) provided arbitration for any discrepancies not resolved through consensus.

For eligible studies, the characteristics extracted included author, title and year of publication, year of study, country, study design, facility, population (diarrhoea, malaria, all admissions, etc), time of enrolment (admission or discharge), number of subjects, age, sex and study eligibility criteria. Outcomes extracted included total number of subjects who died both in-hospital and following discharge, timing and location of postdischarge deaths, follow-up method and losses to follow-up, number of postdischarge rehospitalisations and health seeking, timing of rehospitalisations and health seeking and risk factors for postdischarge mortality. When extracting data on risk factors, the results of multivariate analysis were preferentially extracted over univariate analyses.

\section{Risk of bias}

A formal risk of bias assessment, such as the Newcastle Ottawa Quality Assessment Scale for Cohort Studies, was not conducted since the primary outcome of the rate of postdischarge mortality was not exposure related among included studies. Primary factors leading to potential bias include the per cent follow-up as well as whether inclusion criteria were correctly applied to enrolled subjects, leading to a representative sample of the population. While the former was included in the outcome characteristics, the latter was not defined in any study. Thus, proportion of children successfully followed remains the primary indicator of risk of bias.

\section{Data analysis and outcomes}

Microsoft Excel (Redmond, Washington, USA) was used to compile extracted data. Due to varying populations, risk factors, definitions and types of results (eg, OR, HR), a formal meta-analysis was not deemed possible. Therefore, the analysis was descriptive in nature. The primary outcome was the proportion of discharged subjects who died during the postdischarge period. Secondary outcomes included the proportion of total deaths (in-hospital and postdischarge), which occurred following discharge, as well as risk factors associated with postdischarge mortality. Given that several distinct populations were evaluated, results were reported according to the underlying study population. Studies were grouped according to five underlying populations: (1) all admissions including those for infectious diseases, (2) malnutrition, (3) respiratory infection, (4) diarrhoeal diseases and (5) malaria/anaemia.

\section{RESULTS}

\section{Summary of included articles}

A total of 1238 articles were identified through the systematic searches, with two additional articles identified independently. Of these, 1174 were excluded at the abstract stage and a further 55 were excluded during the full-text screening stage, resulting in 11 eligible studies (figure 1, online supplementary appendix 2). These 11 studies were added to the 13 studies identified prior to 2012 through a similar systematic search, ${ }^{5}$ resulting in a total of 24 included studies (table 2). Studies were grouped according to underlying population. Three studies examined either all admissions or all infectious admissions, five examined malnutrition, seven respiratory infection, three diarrhoeal diseases and six included children with malaria and/or anaemia. Seven randomised controlled trials, 12 prospective cohorts, 2 retrospective cohorts and 3 case-control studies were included. Two studies examined those admitted to a health centre, whereas the remaining 22 were conducted at various types and levels of hospitals. All studies were performed in a single country, and Bangladesh was the only non-African country in which included studies were conducted.

\section{All admissions, including unspecified infectious admissions}

The three studies within this population were conducted between 1991 and 2013 in Guinea-Bissau, Kenya and Uganda, and enrolled between 1307 and 10277 subjects (table 2) ${ }^{8-10}$ Follow-up periods ranged from 6 months to 1 year, with postdischarge mortality ranging from $4.9 \%$ to $8 \%$ (table 3). Two studies reported postdischarge readmission, measured rates between $16.5 \%$ and $17.7 \% .^{9-11}$ Inpatient mortality was recorded by two studies, finding rates of $4.9 \%$ and $15 \% .{ }^{810}$ These same studies recorded that most postdischarge deaths $(67 \%$ and $77 \%)$ occurred outside of the hospital setting. The majority of postdischarge deaths occurred relatively early in the follow-up period, with $63 \%$ occurring within 13 (of 52) weeks in one study and $50 \%$ within 8 (of 24) weeks in the other study. ${ }^{8}{ }^{10}$ Several variables were included in risk factor analyses for postdischarge mortality (table 4 ). Increasing age was shown to be a protective factor in all three studies. Parasitaemia was found to be associated with lower PDM compared with other diagnoses in two studies, with the third study showing lower PDM compared with diarrhoea, anaemia and other less common diagnoses. Bacteraemia, severe or very severe pneumonia, severe malnutrition, meningitis and HIV were all associated with a higher probability of postdischarge death. ${ }^{910}$ In the study by Veirum $e t$ 


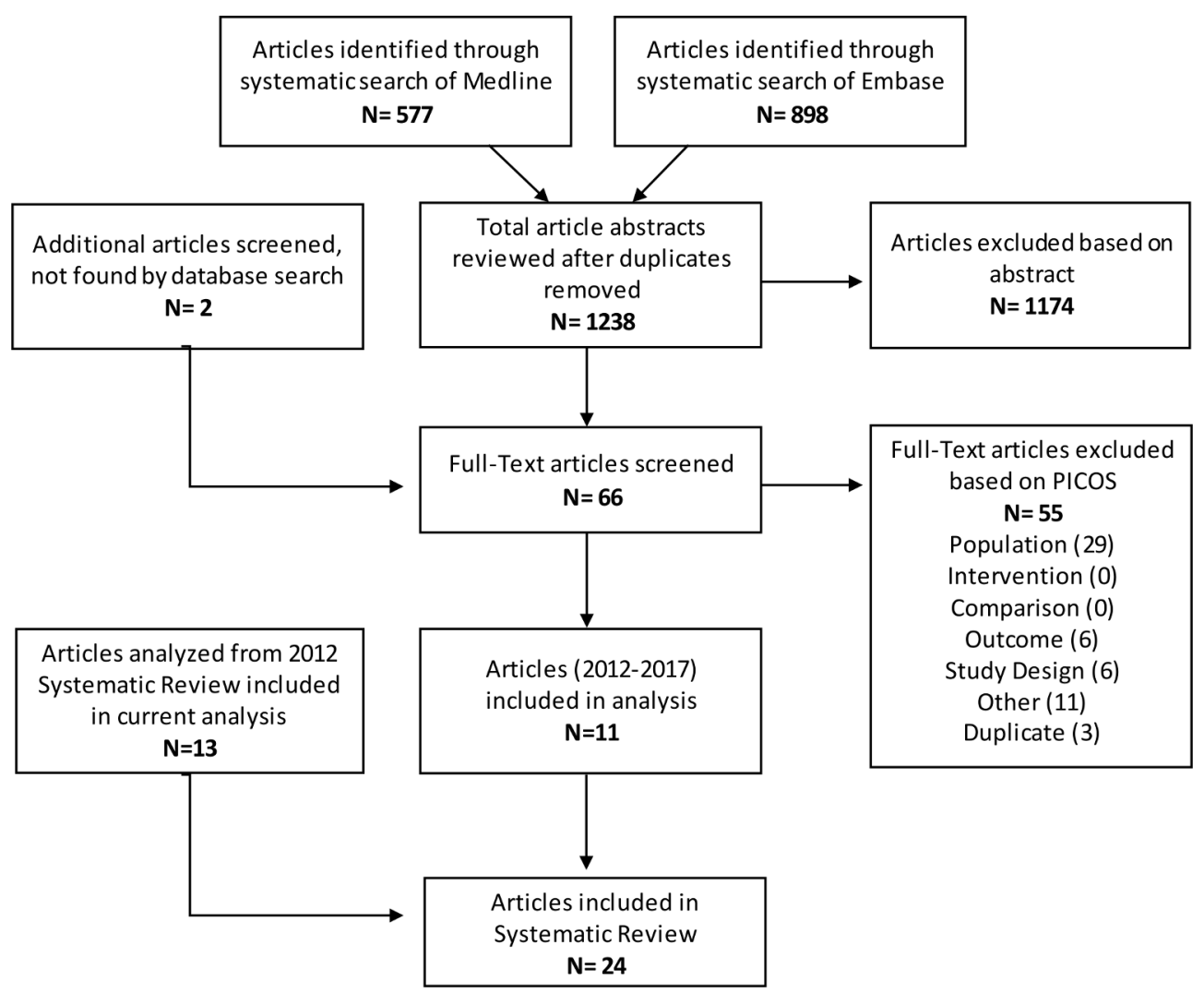

Figure 1 Preferred Reporting Items for Systematic Reviews and Meta-Analyses flow diagram.

al that evaluated discharge against medical advice (AMA), those who left AMA were eight times more likely to die after discharge. ${ }^{8}$ Anthropometric factors (including mid-upper arm circumference (MUAC), weight-for-age, weight-for-height and height-for-age z-scores), hypoxia, respiratory rate, jaundice, hepatomegaly and Blantyre coma scale rating were all associated with a statistically significant increase in the probability of PDM. ${ }^{910}$ Those who had been hospitalised prior to the index admission were also at increased risk for death, with each additional hospitalisation compounding the risk. ${ }^{910}$

\section{Malnutrition}

Five studies focusing on a malnourished population were identified. These studies were conducted in the Democratic Republic of the Congo, Malawi, Bangladesh, Kenya and Uganda between 1970 and 2015 and and enrolled between 171 and 1778 children (table 2). The period of follow-up varied widely in this subpopulation, ranging from 8 weeks to 5 years (table 3). Postdischarge mortality rates were observed to be between $1.8 \%$ and $24 \%$. Where hospital mortality rates were measured $(n=2)$, mortality following discharge was comparable to that observed during hospital admission, with one study reporting an inpatient mortality rate of $23.2 \%$ (24\% after discharge), and a second study of $8.6 \%$ (8.7\% after discharge).${ }^{12} 13$ Three of four studies specified the timing of deaths during the follow-up period with all finding that the majority of deaths following discharge occurred early during the follow-up period (relative to total follow-up).
In one study, $59 \%$ of those who died after discharge did so within 52 weeks (of 5 years), ${ }^{14}$ another found that $44 \%$ died within 13 (of 52) weeks, ${ }^{12}$ and the third study observed $88 \%$ dying within 9 (of 26 ) weeks of discharge. ${ }^{13}$ A study conducted in Bangladesh reporting the location of postdischarge death found that $80 \%$ of deaths occurred at home, while another conducted in Kenya found $53 \%$ occurring in the community. ${ }^{13} 15$ Anthropometric parameters including MUAC, weight-for-age and weight-for-height z-scores were among the highly significant predictors for death postdischarge (table 5).${ }^{12}{ }^{13}$ Age $<12$ months was associated with mortality in one study, but was found not to be significant in another, although wide CIs could not rule out an important effect. ${ }^{12} 13$ Highly significant associations variables included positive HIV status (HR 4.03; 95\% CI 3.08 to 5.25), unknown HIV status (HR 16.90; 95\% CI 12.10 to 23.70) and discharge AMA (HR 4.68; 95\% CI 2.01 to 10.85 ).

\section{Respiratory infection}

Seven studies examining respiratory infections were identified. These included children with a variety of inclusion criteria, including pneumonia, acute lower respiratory tract infection and tuberculosis. Studies were conducted between 1992 and 2014 in the Gambia, Tanzania, Bangladesh, Malawi and Kenya (table 2). Mortality rates postdischarge ranged widely, from $1.3 \%$ to $35 \%$ across the studies. These rates, however, remained consistently comparable to inpatient mortality when both were measured (table 3). As with other populations, mortality 


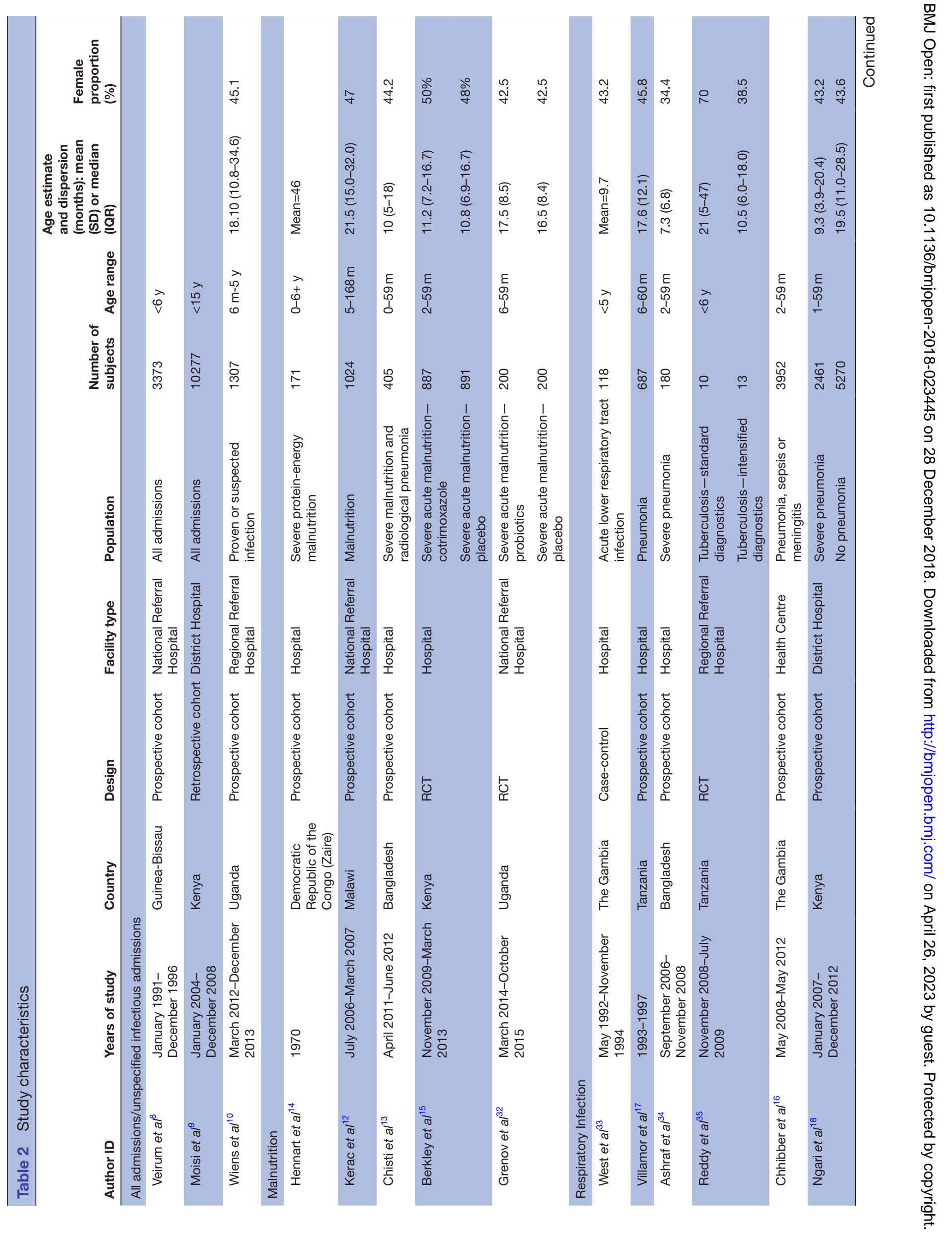




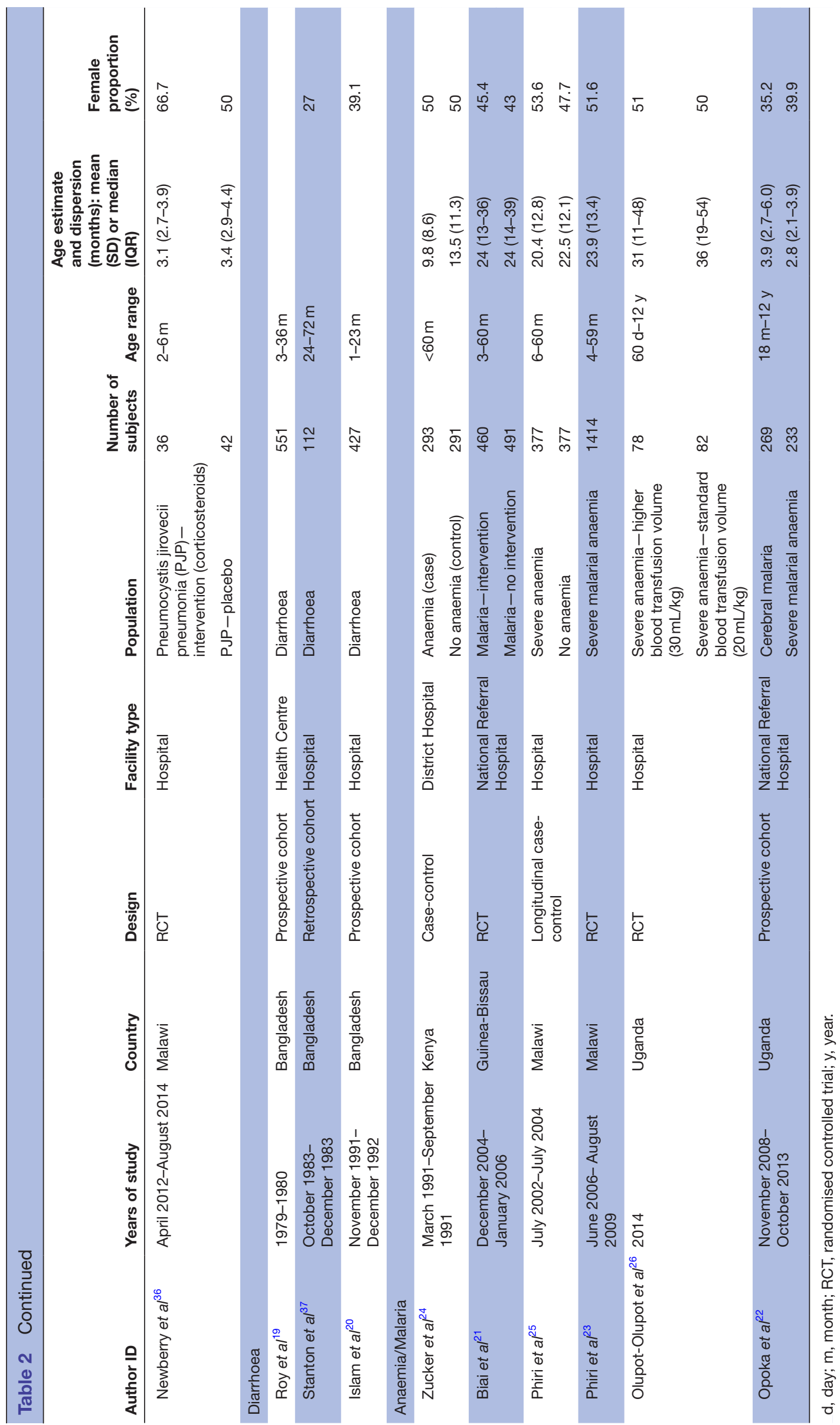




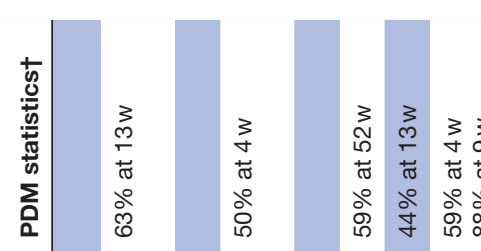

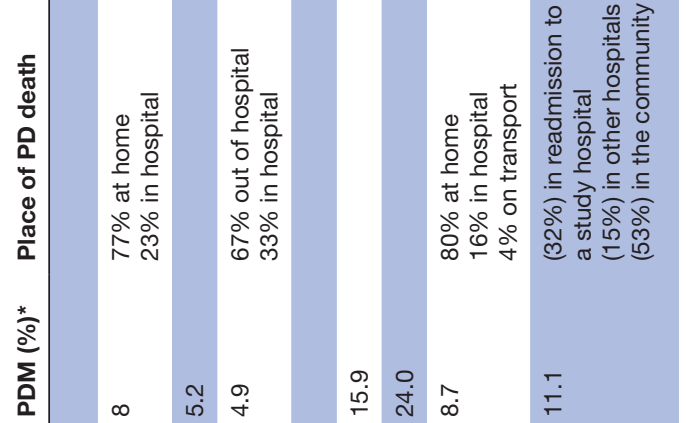

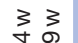

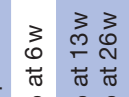

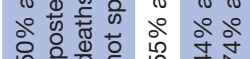

तथ

ลิํํ

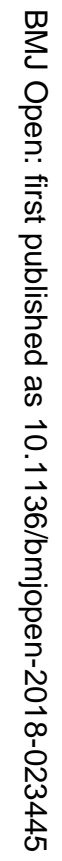

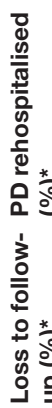

$\dddot{0}$

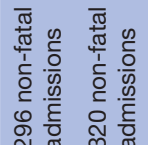

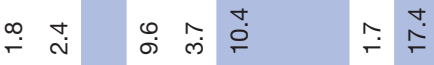

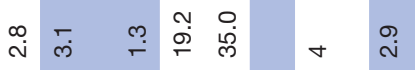

$\stackrel{\leftrightarrow}{6}$

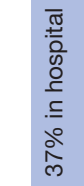

N

○

$\frac{\sqrt{3}}{\mathbb{D}^{3}}$

$\stackrel{2}{\stackrel{7}{ }}$

ஸे

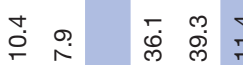

बृ.

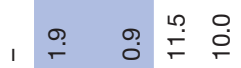

$\stackrel{\infty}{\bullet}$
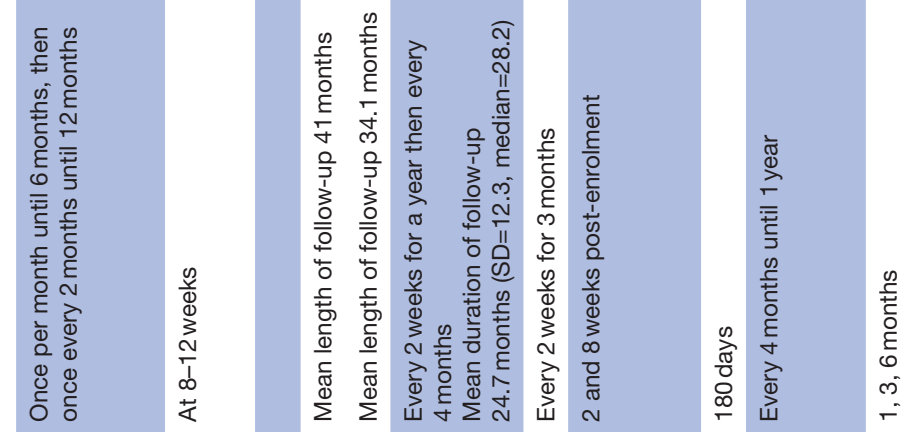

$\stackrel{\text { 옹 }}{\circ}$

¿্

흥

$\frac{0}{\circ}$

훙

룽

홍.

호․

을
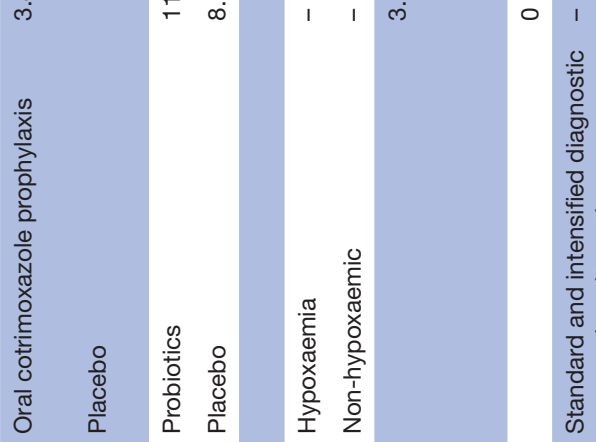

ஓं
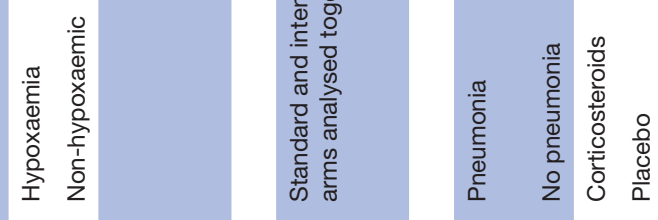

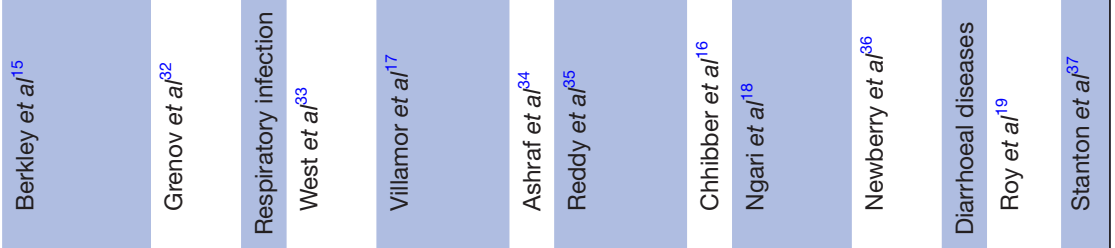

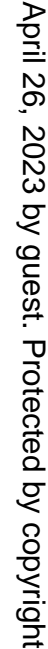




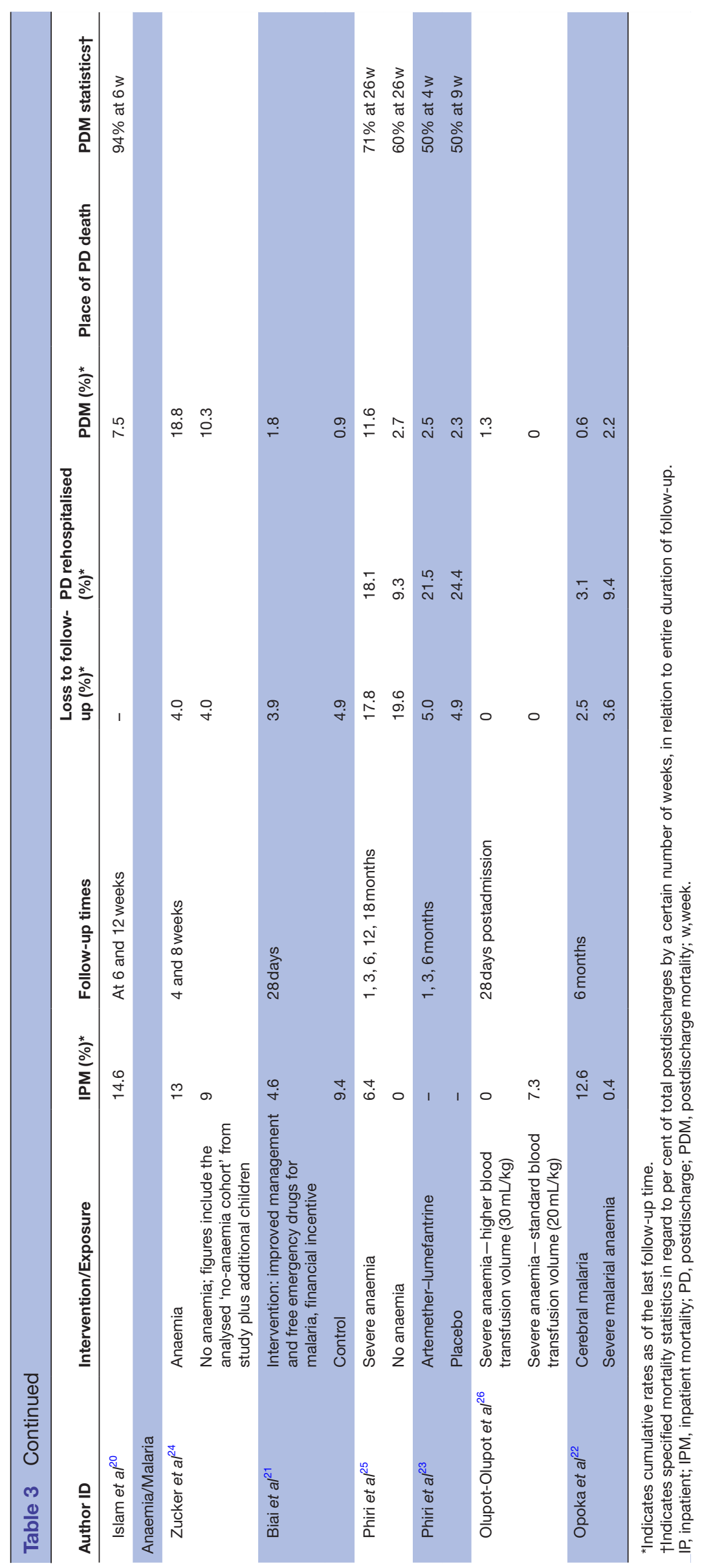

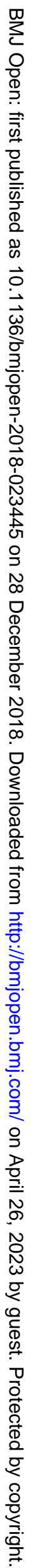




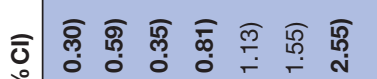

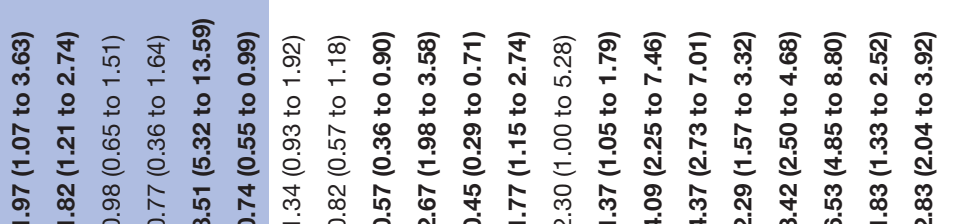

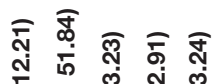

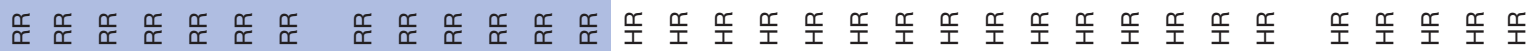

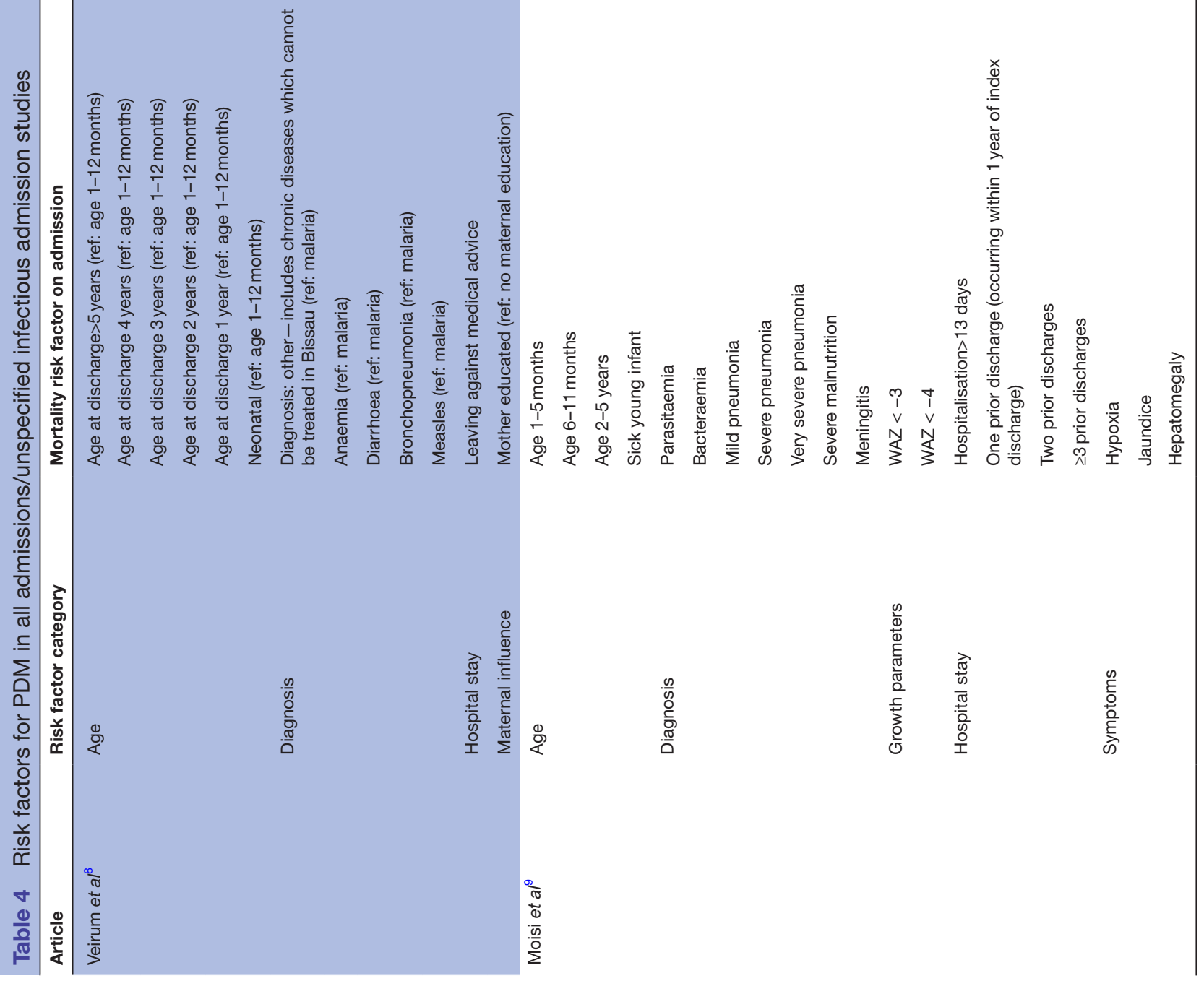




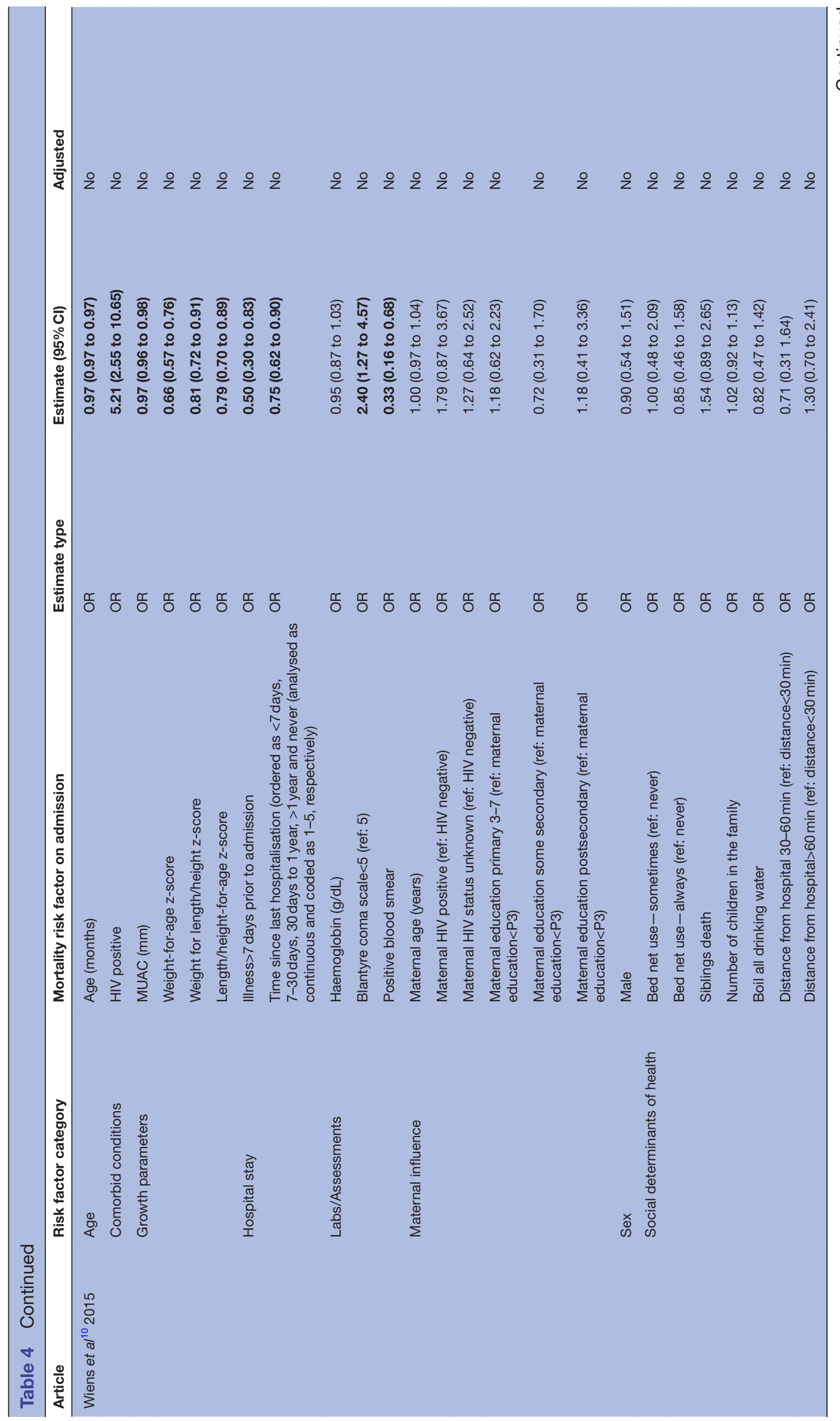


rates generally occurred early during follow-up. A large prospective cohort study by Ngari et al including children aged 1-59 months with severe pneumonia found that $74 \%$ of postdischarge deaths occurred by 26 (of 52) weeks with $63 \%$ occurring outside of the hospital. Chhibber et $a l^{16}$ conducted a study of 3952 children admitted primarily with pneumonia in rural Gambia, and sought to identify specific comorbidities and physiologic factors predictive of mortality after discharge. This study found that physiologic factors, including neck stiffness, oxygen saturation, temperature and haemoglobin concentration were associated with postdischarge mortality. Malnutrition-related variables (clinical malnutrition and low MUAC) were the strongest predictors of postdischarge mortality, producing HRs ranging from 18.4 to 43.7 (table 6). Although individual studies differed in regard to whether risk factors were measured continuously, categorically or dichotomously, it is clear that the directionality of certain risk factors such as low haemoglobin and low MUAC continue to be associated with higher PDM in children admitted for respiratory illness. ${ }^{16-18}$ When examining the timing of mortality, most cases occurred relatively early during follow-up. One study found that $80 \%$ had occurred by 12 months (mean duration of follow-up 24.7 months), ${ }^{17}$ another study had $55 \%$ by 6 (of 26) weeks ${ }^{16}$ and yet another reported $74 \%$ by 26 (of 52) weeks. ${ }^{18}$ Low MUAC, stunting, HIV-positive status, jaundice, low haemoglobin, under 24 months of age and availability of water were significant predictors of postdischarge mortality among children with respiratory illness. ${ }^{1718}$

\section{Diarrhoeal diseases}

Three studies of paediatric patients with diarrhoea conducted between 1979 and 1992 were included, all three of which were conducted in Bangladesh (table 2). Included studies enrolled children aged 1-72 months and found postdischarge death rates of between $2 \%$ and $8 \%$, all being generally comparable to in-hospital rates (table 3). Deaths occurred within the first few weeks after discharge, with one study reporting $52 \%$ by 4 (of 52) weeks, ${ }^{19}$ and a second reporting $94 \%$ of deaths occurring by 6 (of 12) weeks postdischarge. ${ }^{20}$ Significant risk factors for death after discharge identified in this set of studies included young age ( $<6$ months), not having been breast fed, malnutrition (based on Height for age z-score (HAZ) and WAZ scores), low levels of maternal education and immunisation status of the child (table 7).

\section{Anaemia and/or malaria}

Six studies were conducted between 1991 and 2014 in Kenya, Guinea-Bissau, Malawi and Uganda in children with anaemia and/or malaria (table 2). Studies were heterogeneous in their specified populations, including children with various illness severity, with mortality postdischarge ranging from $0.9 \%$ to $18.8 \%$, and with follow-up periods ranging from 1 to 18 months (table 3 ). In the only study looking 
Table 5 Risk factors for PDM in malnutrition studies

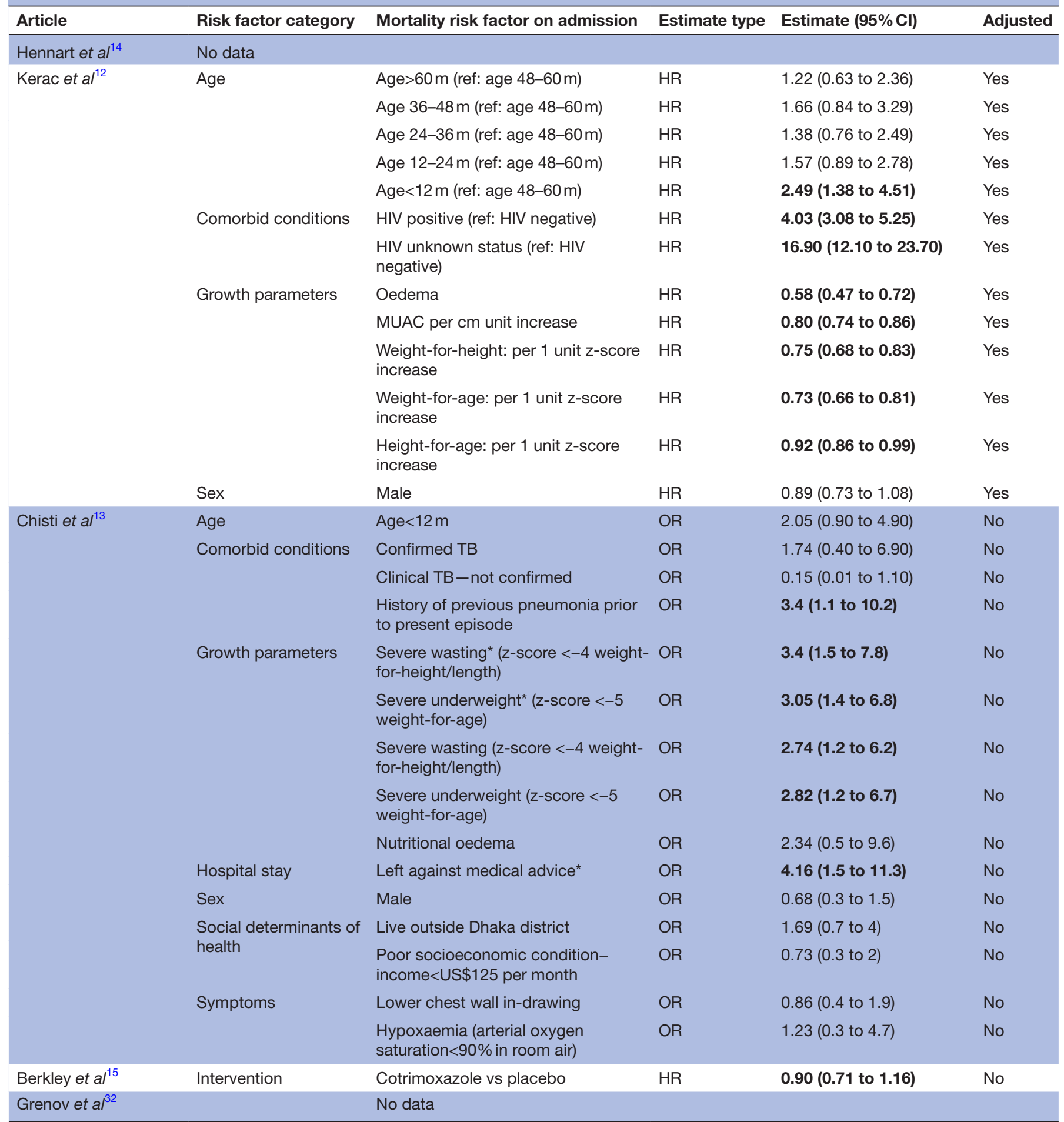

Bolded values are statistically significant.

*Risk factor for mortality assessed on discharge.

$\mathrm{m}$, month; PDM, postdischarge mortality; TB, tuberculosis.

specifically at acute malaria, postdischarge mortality (1.8\% intervention; $0.9 \% \%$ control) was lower than inpatient mortality $(4.6 \%$ intervention; $9.4 \%$ control) over a follow-up period of 28 days. ${ }^{21}$ Another study that followed children with cerebral malaria or severe malarial anaemia for 6 months following discharge reported that although children with cerebral malaria experienced higher inpatient mortality $(13 \%$ compared with $0.4 \%$ ), those with severe malarial anaemia had a higher rate of death after discharge (2.2\% compared with $0.6 \%) .{ }^{22}$ A large study $(\mathrm{n}=1414)$ by Phiri et al examining severe malarial anaemia found high rates of postdischarge readmission (approximately 22\%), with rates of death at approximately 


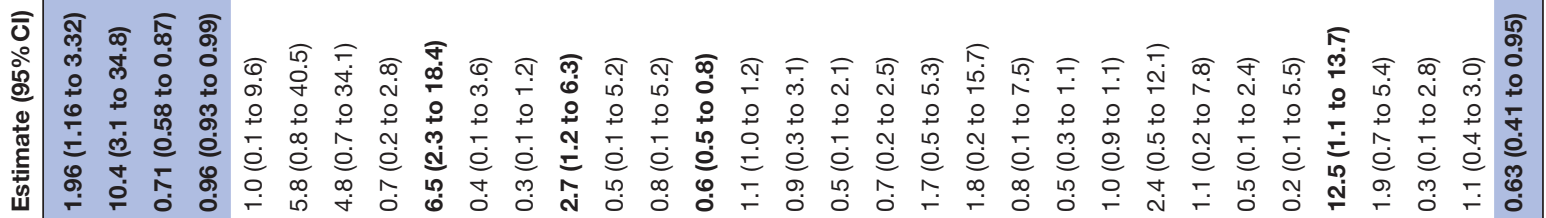

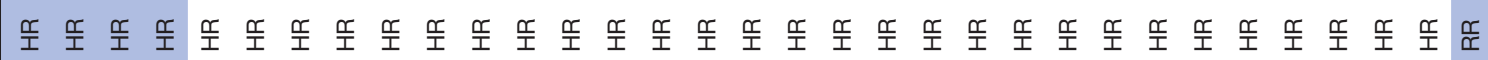
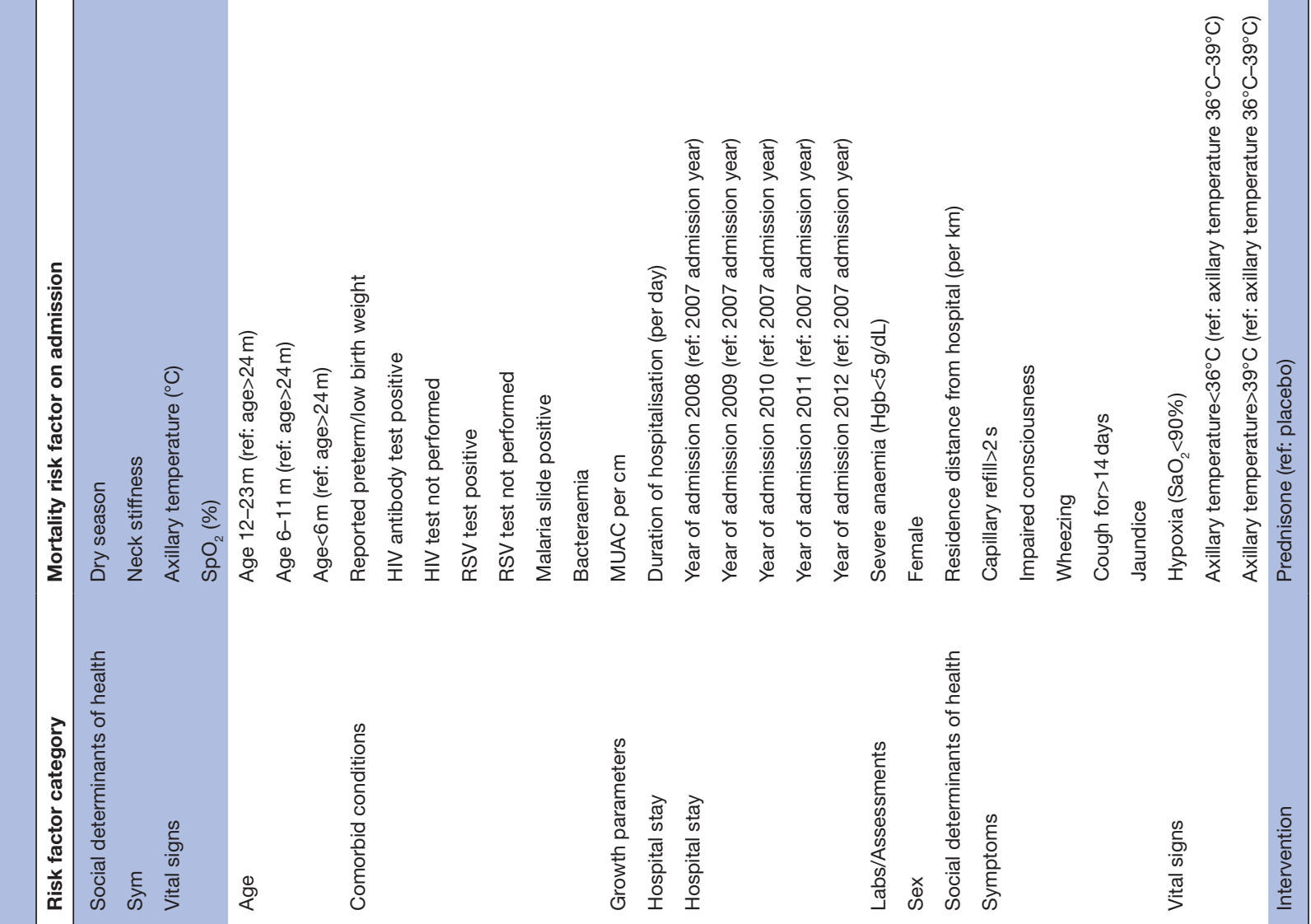
Table 7 Risk factors for PDM in diarrhoea studies

\begin{tabular}{|c|c|c|c|c|c|}
\hline Article & Risk factor category & Mortality risk factor on admission & $\begin{array}{l}\text { Estimate } \\
\text { type }\end{array}$ & Estimate $(95 \% \mathrm{Cl})$ & Adjusted \\
\hline Roy et $a l^{19}$ & No data & & & & \\
\hline Stanton et $\left.a\right|^{37}$ & No data & & & & \\
\hline \multirow[t]{6}{*}{ Islam et al ${ }^{20}$} & Age & Age $<6$ months & $\mathrm{RR}$ & $4.57(2.90$ to 7.18$)$ & Yes \\
\hline & Growth parameters & Weight-for-age median $<60 \%$ & $\mathrm{RR}$ & $1.04(0.57$ to 1.89$)$ & Yes \\
\hline & & Length-for-age median $<85 \%$ & $\mathrm{RR}$ & $2.97(1.43$ to 6.16$)$ & Yes \\
\hline & & No breast feeding & RR & 2.35 (1.44 to 3.84$)$ & Yes \\
\hline & Sex & Female & RR & $1.73(1.14$ to 2.65$)$ & Yes \\
\hline & $\begin{array}{l}\text { Social determinants } \\
\text { of health }\end{array}$ & Immunisation not up-to-date & $\mathrm{RR}$ & $1.36(1.25$ to 1.48$)$ & Yes \\
\hline
\end{tabular}

Bolded values are statistically significant.

PDM, postdischarge mortality; RR, relative risk.

$2.4 \% .^{23}$ Children with anaemia experienced higher rates of inpatient (13\% anaemia; $9 \%$ no anaemia) and postdischarge mortality $(18.8 \%$ anaemia; $10.3 \%$ no anaemia). ${ }^{24}$ In both cohorts, death after discharge was greater than death in-hospital. Rates of readmission to hospital within 18 months were quantified in one study (18.4\% severe anaemia; $9 \%$ no anaemia) and postdischarge mortality rates $(11.6 \%$ anaemia; $2.7 \%$ no anaemia) exceed those of inpatient mortality rates $\left(6.4 \%\right.$ anaemia; $0 \%$ no anaemia). ${ }^{25}$ Although this study had approximately 18\% loss to follow-up, $71 \%$ of anaemic and $60 \%$ of non-anaemic total postdischarge deaths had occurred by 26 (of 78) weeks. ${ }^{25}$ An RCT conducted in Uganda studied the effect of transfusion volume $(30 \mathrm{~mL} / \mathrm{kg}$ vs the standard $20 \mathrm{~mL} /$ $\mathrm{kg}$ ) in severely anaemic children, which showed reduced inpatient mortality rates but no difference for deaths after discharge (table 8) ${ }^{26}$ Rates of death were consistently higher after discharge than in hospital in paediatric patients presenting with

Table 8 Risk factors for PDM in anaemia/malaria studies

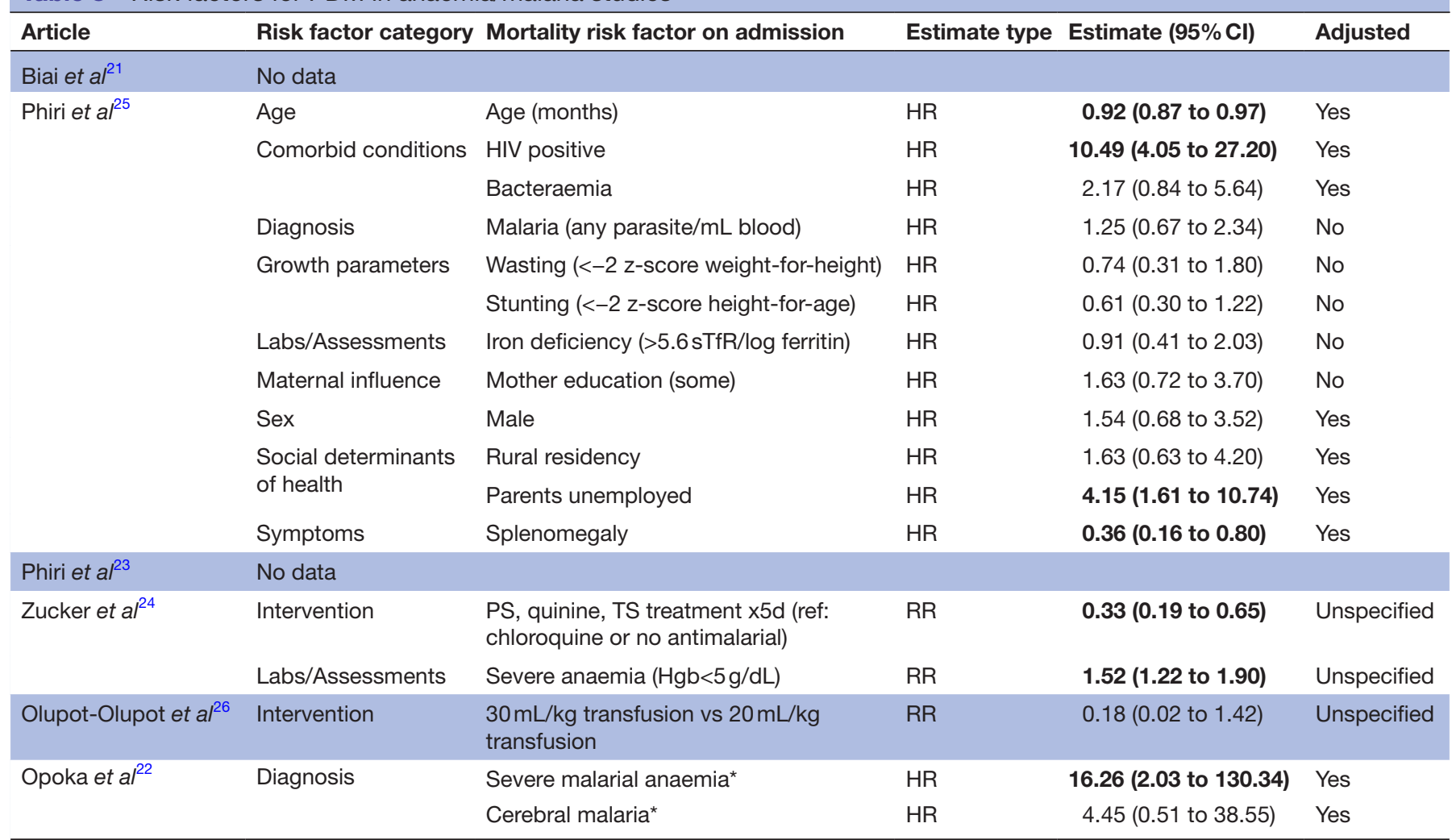

${ }^{*}$ Risk factor for readmission.

$\mathrm{Hgb}$, haemoglobin; PDM, postdischarge mortality; RR, relative risk. 
malaria and/or anaemia. Of risk factors identified throughout these studies, severe anaemia was found to be highly significant for postdischarge death ${ }^{24}$ and readmission to the hospital. ${ }^{22}$ HIV status profoundly influenced mortality, with a HR of 10.49 (95\% CI 4.05 to 27.20) for death postdischarge in children who tested positive. $^{25}$

\section{DISCUSSION}

Twenty-four studies examining postdischarge mortality in paediatric populations in developing countries were included in this systematic review, together substantiating the significant and unaddressed challenge continuing to plague children around the world. Significant heterogeneity in study characteristics was noted, within inclusion criteria, study design, length of follow-up, interventions (if any), risk factors and risk factor definitions. Studies were conducted primarily in African countries, and examined a variety of populations, including all admissions, infectious disease admissions, malnutrition, respiratory infections, diarrhoea, malaria and anaemia. Studies examining anaemia and/or malaria had the lowest PDM rates, while those of malnutrition and respiratory infections had the highest. Results from the studies identified through the updated search generally reflected the results from the earlier systematic review; rates of postdischarge mortality continued to be high and comparable to (sometimes exceeding) in-hospital mortality, with most postdischarge deaths occurring at home. ${ }^{5}$ With so many deaths occurring after discharge, it is critical that effective interventions be developed and evaluated as a means to addressing this neglected cause of childhood deaths. Furthermore, no analysis of cause for death postdischarge was identified within any of the reviewed studies, highlighting this as an important area for further research.

When reported, over two-thirds of postdischarge deaths were noted to occur outside of the hospital, generally at home. In order to develop interventions to reduce the burden of PDM, an understanding of circumstances and barriers to care following discharge is of utmost importance. In a recent qualitative study, mothers of children who died postdischarge identified barriers to seeking care prior to their child's death; barriers included lack of access to health facilities and services, poor healthseeking behaviour, finances, transportation and a lack of recognition of symptoms and perceptions of recovery in children recently discharged even in the midst of persisting illness. ${ }^{27}$ Additional factors that contribute to poor socioeconomic conditions may relate to deaths after discharge, as they further disadvantage children and families. Socioeconomically disadvantaged children continue to be served by health sectors that are poorly resourced and lack the resilience to be able to deal with large numbers of patients seen every day. Follow-up care after initial hospitalisation is an important and yet largely ignored aspect of comprehensive healthcare in both developing countries. ${ }^{2}$ With so few patients returning to the healthcare system after discharge, identifying and understanding the barriers and targeted interventions required to enhance outcomes must be initiated during the original hospitalisation.

Risk factors consistently identified across all types of infectious admissions as highly associated with postdischarge mortality included HIV status, young age, pneumonia, malnutrition, anthropometric factors, hypoxia, anaemia, leaving the hospital against medical advice and previous hospitalisations. An important observation, therefore, is that regardless of the underlying infectious aetiology, certain risk factors consistently identify vulnerability. These observations suggest that vertical, diseasebased, approaches to addressing postdischarge mortality are likely to be ineffective in comparison to simple, broadly applicable interventions. Specific illness (ie, pneumonia, diarrhoea, malaria) are often both difficult to differentiate clinically and often co-exist, especially in children in low-resource settings. ${ }^{28}$ Sepsis, therefore, as the final common pathway for the majority of infectious disease-related deaths, may be a helpful framework within which to explore paediatric postdischarge mortality and to develop interventions. Instead of focusing on a specific body system or infectious agent, pragmatic interventions towards time-sensitive treatment can be focused towards sepsis as the overarching syndrome, increasing the potential for impactful results. ${ }^{28}$ The Integrated Management of Childhood Illness (IMCI) pocketbook by the WHO uses a similar approach through their identification of danger signs and treatments as opposed to individual diseases. Addressing sepsis through clinical management is an important component in the reduction of preventable childhood death, requiring sustained efforts by the global community including healthcare providers, patients, pharmaceutical companies and policy makers if large-scale change is to occur. ${ }^{29}$

While knowledge of risk factors alone has only moderate utility in the identification of vulnerable children, the development of robust prediction models can provide a more reliable means of risk evaluation. In resource-limited environments, the use of prediction modelling is appealing, especially in relation to interventions aimed at improving postdischarge outcomes. A recent proof-ofconcept study found that a simple discharge intervention including education and routine postdischarge follow-up could substantially improve postdischarge health seeking and health outcomes. ${ }^{30}$ Such approaches, if focused primarily on the most vulnerable children, can ensure that limited resources are most effectively used and have the highest possible level of cost-effectiveness.

This systematic review is subject to several important limitations. First, it is possible that some relevant articles may not have been identified through the systematic search. Although the search was comprehensive, including both Medline and EMBASE, no MeSH/Emtree terms currently exist for postdischarge mortality and even so, many studies measure postdischarge mortality as a secondary end point. A further limitation of this review 
is that the studies included were predominantly based in African countries. Therefore, these results may not be as applicable to countries outside of this setting. This highlights the continued need for ongoing research in resource-poor settings both within and outside of Africa. Significant heterogeneity in duration of follow-up, as well as when postdischarge mortality was assessed, was noted between the studies, potentially leading to a decreased ability to compare mortality rates. Many studies included in this review had high losses to follow-up (ranging between $0 \%$ and $39.3 \%$ ), and very few were conducted prospectively with the stated intent of exploring postdischarge mortality. Studies with significant attrition due to follow-up likely underestimate the true rate of postdischarge mortality as these losses undoubtedly represent a more vulnerable population. While one study focused on barriers to care following discharge among those children who died in the community, one important remaining gap is that studies did not evaluate the causes of postdischarge mortality, which is difficult to measure given that most deaths occur in the community. ${ }^{1027}$ The ongoing, multicountry, Childhood Acute Illness and Nutrition (CHAIN) network, is attempting to understand the specific reasons for deaths postdischarge among malnourished children. ${ }^{31}$ It is through contributions such as this that further interventions can be developed and implemented that target the specific and causal factors affecting paediatric mortality rates in developing countries.

\section{CONCLUSIONS}

In conclusion, the studies identified emphasise the significant burden of postdischarge mortality in countries where overextended and resource-limited health systems serve millions of socioeconomically disadvantaged children. The scale of this burden continues to be under-recognised, in part due to the inability of health systems to observe patient outcomes after discharge. Addressing these issues with specific regard to the identification of vulnerable children, and the development of effective postdischarge interventions, will be an essential component towards the achievement of the child mortality targets of the SDGs.

\section{Author affiliations}

${ }^{1}$ College of Nursing, University of Saskatchewan, Saskatoon, Saskatchewan, Canada

${ }^{2}$ Department of Medicine, University of North Carolina, Raleigh, North Carolina, USA ${ }^{3}$ Department of Pediatrics, University of British Columbia, Vancouver, British Columbia, Canada

${ }^{4}$ Center for International Child Health, BC Children's Hospital, Vancouver, British Columbia, Canada

${ }^{5}$ Anesthesiology, Pharmacology and Therapeutics, University of British Columbia, Vancouver, Canada

${ }^{6}$ Division of Global Health, Massachusetts General Hospital, Boston, Massachusetts, USA

${ }^{7}$ Maternal, Newborn and Child Health Institute, Mbarara University of Science and Technology, Mbarara, Uganda

${ }^{8}$ Faculty of Medicine, Mbarara University of Science and Technology, Mbarara, Uganda
Contributors BN: conceptualised and designed the review, carried out article analysis, drafted the initial manuscript, critically reviewed and revised the manuscript and approved the final manuscript as submitted. LE: contributed to analysis, reviewed and revised for important intellectual content and approved the final manuscript as submitted. NK: contributed to conception and design, interpretation of data and reviewed and revised the manuscript for important intellectual content and approved the final manuscript as submitted. JMA: contributed to conception and design, interpretation of data, reviewed and revised the manuscript for important intellectual content and approved the final manuscript as submitted. PPM: contributed to interpretation of data, reviewed and revised the manuscript for important intellectual content and approved the final manuscript as submitted. JK: contributed to interpretation of data, reviewed and revised the manuscript and approved the final manuscript as submitted. SF-K: contributed to interpretation of data, review and revision of manuscript for important intellectual content and approved the final manuscript as submitted. EK: contributed to interpretation of data, review and revision for intellectual content and revised the manuscript and approved the final manuscript as submitted. MOW: conceptualised and designed the review, coordinated and supervised analysis, critically reviewed and revised the manuscript and approved the final manuscript as submitted. All authors approved the final manuscript as submitted and agree to be accountable for all aspects of the work.

Funding The authors have not declared a specific grant for this research from any funding agency in the public, commercial or not-for-profit sectors.

Competing interests None declared.

Patient consent Not required.

Provenance and peer review Not commissioned; externally peer reviewed.

Data sharing statement There is no additional unpublished data from this study, as it is a systematic literature review. Any additional information is contained within the submitted appendices.

Open access This is an open access article distributed in accordance with the Creative Commons Attribution Non Commercial (CC BY-NC 4.0) license, which permits others to distribute, remix, adapt, build upon this work non-commercially, and license their derivative works on different terms, provided the original work is properly cited, appropriate credit is given, any changes made indicated, and the use is non-commercial. See: http://creativecommons.org/licenses/by-nc/4.0/.

\section{REFERENCES}

1. Transforming our world: the 2030 Agenda for Sustainable Development. New York: United Nations General Assembly, 2015.

2. Wiens MO, Kissoon N, Kabakyenga J. Smart discharges to address a neglected epidemic in sepsis. JAMA pediatrics 2018;172:213-4.

3. Reinhart K, Daniels R, Kissoon N, et al. Recognizing sepsis as a global health priority - a WHO resolution. $N$ Engl $\mathrm{J} \mathrm{Med}$ 2017;377:414-7.

4. Kissoon N, Reinhart K, Daniels R, et al. Sepsis in children: global implications of the World Health Assembly Resolution on Sepsis. Pediatr Crit Care Med 2017;18:e625-7.

5. Wiens MO, Pawluk S, Kissoon N, et al. Pediatric post-discharge mortality in resource poor countries: a systematic review. PLOS One 2013;8:e66698.

6. Jahan S. Overview: human development report 2016: human development for everyone. New York, NY: United Nations Development Programme, 2016.

7. Klugman J. Human development report 2011. New York: United Nations Development Program, 2011.

8. Veirum JE, Sodeman M, Biai S, et al. Increased mortality in the year following discharge from a paediatric ward in Bissau, Guinea-Bissau. Acta Paediatr 2007;96:1832-8.

9. Moïsi JC, Gatakaa H, Berkley JA, et al. Excess child mortality after discharge from hospital in Kilifi, Kenya: a retrospective cohort analysis. Bull World Health Organ 2011;89:725-32.

10. Wiens MO, Kumbakumba E, Larson CP, et al. Postdischarge mortality in children with acute infectious diseases: derivation of postdischarge mortality prediction models. BMJ Open 2015;5:e009449.

11 Wiens MO. Childhood mortality from acute infectious diseases in Uganda: Studies in sepsis and post-discharge mortality: University of British Columbia, 2015.

12. Kerac M, Bunn J, Chagaluka G, et al. Follow-up of post-discharge growth and mortality after treatment for severe acute malnutrition (FuSAM study): a prospective cohort study. PLoS One 2014;9:1-10. 
13. Chisti MJ, Graham SM, Duke T, et al. Post-discharge mortality in children with severe malnutrition and pneumonia in Bangladesh. PLoS One 2014;9:e107663.

14. Hennart P, Beghin D, Bossuyt M. Long-term follow-up of severe protein-energy malnutrition in Eastern Zaïre. $J$ Trop Pediatr 1987;33:10-12.

15. Berkley JA, Ngari M, Thitiri J, et al. Daily co-trimoxazole prophylaxis to prevent mortality in children with complicated severe acute malnutrition: a multicentre, double-blind, randomised placebocontrolled trial. Lancet Glob Health 2016;4:e464-73.

16. Chhibber AV, Hill PC, Jafali J, et al. Child mortality after discharge from a health facility following suspected pneumonia, meningitis or septicaemia in rural gambia: a cohort study. PLoS One 2015;10:e0137095.

17. Villamor E, Misegades L, Fataki MR, et al. Child mortality in relation to HIV infection, nutritional status, and socio-economic background. Int J Epidemiol 2005;34:61-8.

18. Ngari MM, Fegan G, Mwangome MK, et al. Mortality after inpatient treatment for severe pneumonia in children: a cohort study. Paediatr Perinat Epidemiol 2017;31:233-42.

19. Roy SK, Chowdhury AK, Rahaman MM. Excess mortality among children discharged from hospital after treatment for diarrhoea in rural Bangladesh. Br Med J 1983;287:1097-9.

20. Islam MA, Rahman MM, Mahalanabis D, et al. Death in a diarrhoeal cohort of infants and young children soon after discharge from hospital: risk factors and causes by verbal autopsy. J Trop Pediatr 1996;42:342-7.

21. Biai S, Rodrigues A, Gomes M, et al. Reduced in-hospital mortality after improved management of children under 5 years admitted to hospital with malaria: randomised trial. BMJ 2007;335:862.

22. Opoka RO, Hamre KES, Brand N, et al. High postdischarge morbidity in ugandan children with severe malarial anemia or cerebral malaria. $J$ Pediatric Infect Dis Soc 2016:64:piw060.

23. Phiri $\mathrm{K}$, Esan $\mathrm{M}$, van Hensbroek $\mathrm{MB}$, et al. Intermittent preventive therapy for malaria with monthly artemether-lumefantrine for the post-discharge management of severe anaemia in children aged 4-59 months in southern Malawi: a multicentre, randomised, placebo-controlled trial. Lancet Infect Dis 2012;12:191-200.

24. Zucker JR, Lackritz EM, Ruebush TK, et al. Childhood mortality during and after hospitalization in western Kenya: effect of malaria treatment regimens. Am J Trop Med Hyg 1996;55:655-60.
25. Phiri KS, Calis JC, Faragher B, et al. Long term outcome of severe anaemia in Malawian children. PLoS One 2008;3:e2903.

26. Olupot-Olupot P, Engoru C, Thompson J, et al. Phase II trial of standard versus increased transfusion volume in Ugandan children with acute severe anemia. BMC Med 2014;12:67.

27. English L, Kumbakumba E, Larson CP, et al. Pediatric out-of-hospital deaths following hospital discharge: a mixed-methods study. Afr Health Sci 2016;16:883-91.

28. Kissoon N, Carapetis J. Pediatric sepsis in the developing world. $J$ Infect 2015;71:S21-6.

29. Dugani S, Laxminarayan R, Kissoon N. The quadruple burden of sepsis. CMAJ 2017;189:E1128-29.

30. Wiens MO, Kumbakumba E, Larson CP, et al. Scheduled follow-up referrals and simple prevention kits including counseling to improve post-discharge outcomes among children in uganda: a proof-ofconcept study. Glob Health Sci Pract 2016;4:422-34.

31. The Childhood Acute Illness \& Nutrition Network. 2017. http://www. chainnetwork.org/about-us/

32. Grenov B, Namusoke H, Lanyero B, et al. Effect of probiotics on diarrhea in children with severe acute malnutrition: a randomized controlled study in Uganda. J Pediatr Gastroenterol Nutr 2017;64:396-403.

33. West TE, Goetghebuer T, Milligan P, et al. Long-term morbidity and mortality following hypoxaemic lower respiratory tract infection in Gambian children. Bull World Health Organ 1999;77:144-8.

34. Ashraf $\mathrm{H}$, Alam NH, Chisti MJ, et al. Observational follow-up study following two cohorts of children with severe pneumonia after discharge from day care clinic/hospital in Dhaka, Bangladesh. BMJ Open 2012;2:e000961.

35. Reddy EA, Njau BN, Morpeth SC, et al. A randomized controlled trial of standard versus intensified tuberculosis diagnostics on treatment decisions by physicians in Northern Tanzania. BMC Infect Dis 2014;14:89.

36. Newberry L, O'Hare B, Kennedy N, et al. Early use of corticosteroids in infants with a clinical diagnosis of Pneumocystis jiroveci pneumonia in Malawi: a double-blind, randomised clinical trial. Paediatr Int Child Health 2017;37:121-8.

37. Stanton B, Clemens J, Khair T, et al. Follow-up of children discharged from hospital after treatment for diarrhoea in urban Bangladesh. Trop Geogr Med 1986;38:113-8. 